\title{
Microwave Ion Source for Low Charge State Ion Production*
}

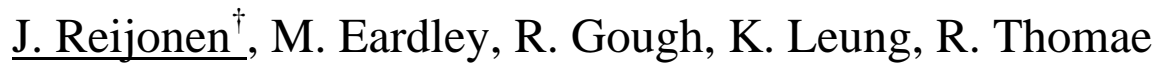 \\ Lawrence Berkeley National Laboratory, 1 Cyclotron Road, MS 5-119, Berkeley, CA 94720, USA
}

\begin{abstract}
The Plasma and Ion Source Technology Group at LBNL have developed a microwave ion source. The source consists of a stainless-steel plasma chamber, a permanent-magnet dipole structure and a coaxial microwave feed. Measurements were carried out to characterize the plasma and the ion beam produced in the ion source. These measurements included current density, charge state distribution, gas efficiency and accelerated beam emittance measurements. Using a computer controlled data acquisition system a new method of determining the saturation ion current was developed. Current density of 3-6 mA/ $\mathrm{cm}^{2}$ was measured with the source operating at the over dense mode. The highest measured charge-states were $\mathrm{Ar}^{5+}, \mathrm{O}^{3+}$ and $\mathrm{Xe}^{7+}$. Gas efficiency was measured using a calibrated argon leak. Depending on the source pressure and discharge power, more than $20 \%$ total gas efficiency was achieved. The emittance of the ion beam was measured by using a pepper-pot device. Certain spread was noticed in the beam emittance in the perpendicular direction to the source dipole field. For the parallel direction to the magnetic field, the normalized $r r$ ' emittance of $0.03 \pi$-mm-mrad at $13 \mathrm{kV}$ of acceleration voltage and beam exit aperture of 3-mm-in-diameter was measured. This compares relatively well with the simulated value of $4 \mathrm{rms}$, normalized emittance value of $0.024 \pi$-mmmrad.
\end{abstract}

\section{INTRODUCTION}

Single to low charge state ion beams with good beam quality and ion sources with good gas efficiency are needed in various applications, ranging from radioactive ion beam production to high energy ion implanters. A simple permanent-magnet microwave ion source has been developed to fulfill the various requirements.

\section{SOURCE DESIGN}

The plasma chamber of the microwave ion source was made from stainless-steel. Three vacuum feedthrough ports were designed: one to the opposite side of the extraction and two facing each other in the middle of the magnet-rings. The two facing ports were designed to be different in length. This enabled one to test different lengths of the coaxial antenna inside the source. The other two ports were used for gas feed and for a window port.
The magnets were attached around the source using an iron yoke. They were floating around the source chamber so that the cooling air was flowing between the source body and the magnets. This arrangement maintained the temperature of the magnets reasonably low. The magnet rings were constructed from a $\mathrm{SmCo}$ magnet blocks measuring $1.3 \mathrm{~cm}, 2.5 \mathrm{~cm}$ and $3.8 \mathrm{~cm}$ in width, height and depth respectively. To have a ring configuration, using bar magnets, the bar ends close to the feed-trough port were touching each other, but the other ends of the magnet bars were separated. To achieve a fairly uniform field, the magnet poles of the opposite sides were rotated slightly so that the magnets in the poles did not face each other. In figure 1 a schematic drawing of the micro-wave ion source is shown.

Initially the magnetic field $\left(B_{d i p}(d)\right)$ using a yoke, as a function of the distance between the poles was approximately given by

\footnotetext{
* This Work was supported by Department of Energy under Contract No. DE-AC03-76SF00098

† email: jreijonen@lbl.gov
} 


$$
B_{\text {dip }}(d)=B(d)+B(d-g)
$$

where $\mathrm{d}$ is the position between the magnets in the dipole, $\mathrm{g}$ is the distance between the magnet poles and $\mathrm{B}(\mathrm{d})$ is given by[1]

$$
\begin{aligned}
& B(d)=\frac{B_{r}}{\pi}\left(a \tan \left[\frac{d+2 l}{t w} \sqrt{t^{2}+w^{2}+(d+2 l)^{2}}\right]-\right. \\
& \left.a \tan \left[\frac{d}{t w} \sqrt{t^{2}+w^{2}+d^{2}}\right]\right)
\end{aligned}
$$

where the $2 t, 2 \mathrm{w}, 1$ is the width, length and height of the magnet bars.

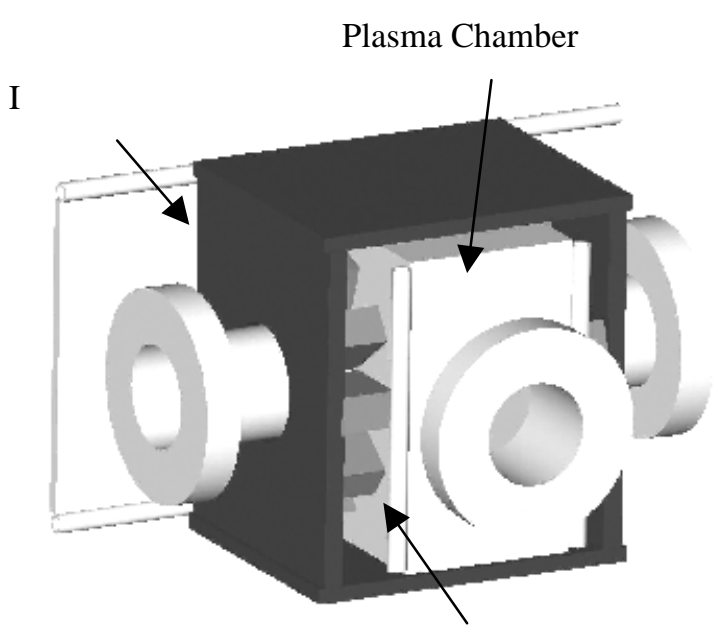

FIGURE 1. The stainless steel ion source body with the dipole ring magnets and the iron yoke. The yoke was strapped around the plasma chamber using (non-magnetic) aluminum strips. The yoke halves are not touching each other. The magnet poles were made to be movable, so that the distance between the magnets was adjustable.

The estimated radial magnetic field values, was relatively close to the measured values as can be seen from figure 2. Also the axial field was measured in different positions between the poles. The axial field measurements are shown in figure 3.

A commercial $2.45 \mathrm{GHz}$ microwave generator was used. The maximum output of the generator is 1000 $\mathrm{W}$. The microwave generator and the microwave injection system consist of a magnetron coupled to a WR-284 size wave-guide. The wave guide was equipped with a three stub tuner. Tuning was optimized by maximizing the output current of the source. After a Teflon vacuum window, the microwave was fed into the source using a coaxial line[2].

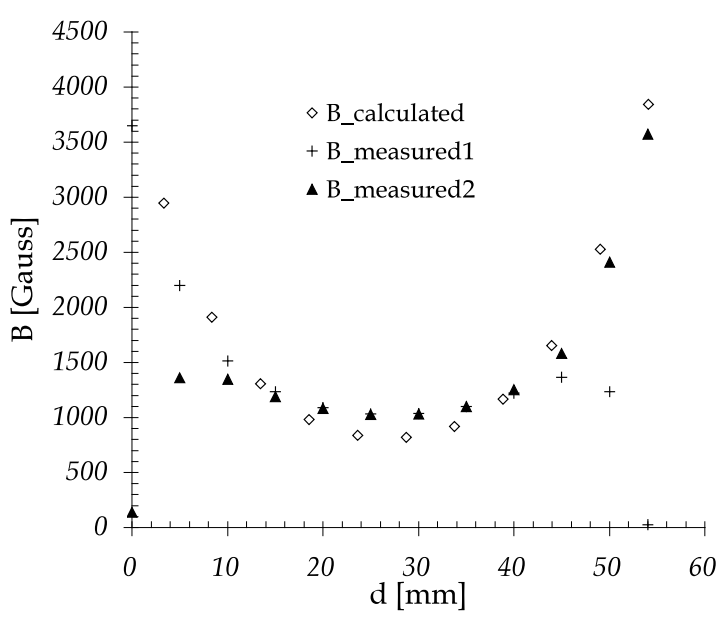

FIGURE 2. The radial magnetic field of the dipole with the iron yoke. The calculated value is compared to the measured value from two places of the dipole, from the top of the magnet to the space between the magnet (B_measured1) and from the place between the magnets to the top of the magnet. The calculated value does not take into account the continues row of magnets, rather it calculates the field produced by pair of 'yoked' magnets. To compensate for the error, the width of the magnet is $1.9 \mathrm{~cm}$ in the calculation.

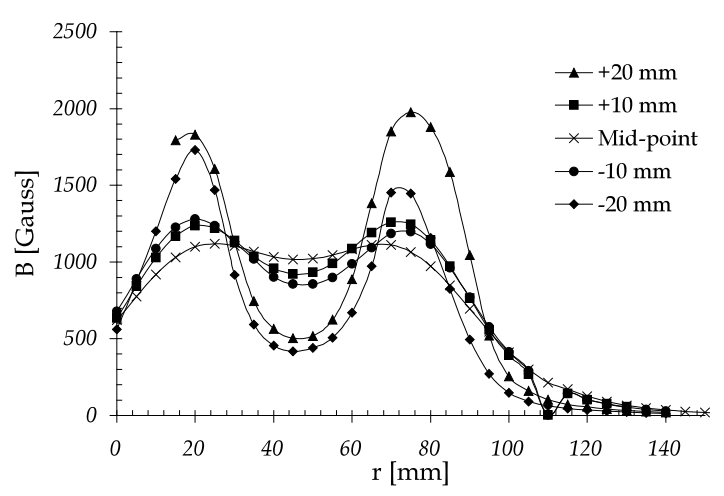

FIGURE 3. The Hall-probe measurements of the microwave source axial magnetic field from the extraction $(0 \mathrm{~mm})$ to the back of the source. The mid-point measurement is performed in the middle of the magnets. (+) and (-)corresponds to the direction toward the middle of the magnet block and the direction toward the space between two blocks respectively. The dipole field at the extraction is approximately 500 Gauss. 
A diode, or two-electrode extraction system was used in the experiments. The configuration was simulated by using the computational code IGUN[3]. A sample simulation run is shown in figure 4 . The plasma electrode is at the source potential and the extractor electrode is at ground potential. The beam exit aperture is $3 \mathrm{~mm}$ in diameter, the aperture in the extractor is $7 \mathrm{~mm}$. The plasma electrode is a double curvature type for minimized aberrations [4].

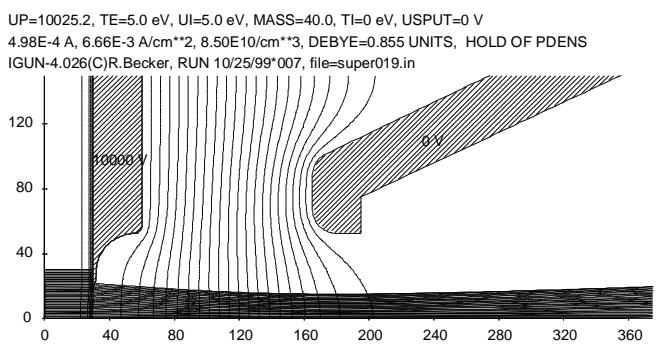

FIGURE 4. The extraction system for the microwave source, simulated by IGUN. The extraction was designed for argon beam at energies $10-15 \mathrm{kV}$. The double curvature plasma electrode provides nearly aberration free beam formation. The ion temperature is zero in this simulation.

\section{MEASUREMENTS}

\section{A. Current Density}

A faraday cup with secondary emission electron suppression is used in both the total beam current and in the charge-state distribution measurements. The current was measured using a computer controlled data acquisition system. A method was developed to determine the saturation current as a function of the acceleration voltage. The saturation point can be obtained by fitting two linear functions to the current/voltage data: one for the fast rising slope part of the data and another to the saturation part of the data. The crossing of those fits gives the saturation current and the corresponding voltage.

The fitting method is fairly cumbersome, so usually the local maximum of the beam perveance is taken as the saturation point. This method unfortunately gives only an estimation of the saturation current, which is smaller than the one obtained with the linear-fit method. Another method was also developed. In that method, the value of the acceleration voltage power supply's leak current was used. The leak current consists of two components: the extracted ion current and of the back streaming electrons. These secondary electrons are generated, either from the ionization of the rest gas or from the secondary electron emission, when the beam hits to a electrode or a faraday cup. The leak current is the sum of both of the electron and ion currents. At low voltage the leak current consists mostly of the back streaming secondary electron current. When the beam converges in the extraction gap, due to the higher voltage, it's size gets smaller and eventually it passes through the extractor electrode. Then the amount of the back streaming electrons will decrease rapidly and the leak current will reach the minimum. This point is approximately equal to the saturation point of the extracted ion current. After this point the leak current will grow mainly with the growing ion current and the back streaming electron current due to the ionization of the rest gas in the acceleration gap. The ratio of the ion current to the leak current of the acceleration power supply has a maximum at close to the saturation point. The perveance method and the ratio-method are shown in figure 5 .
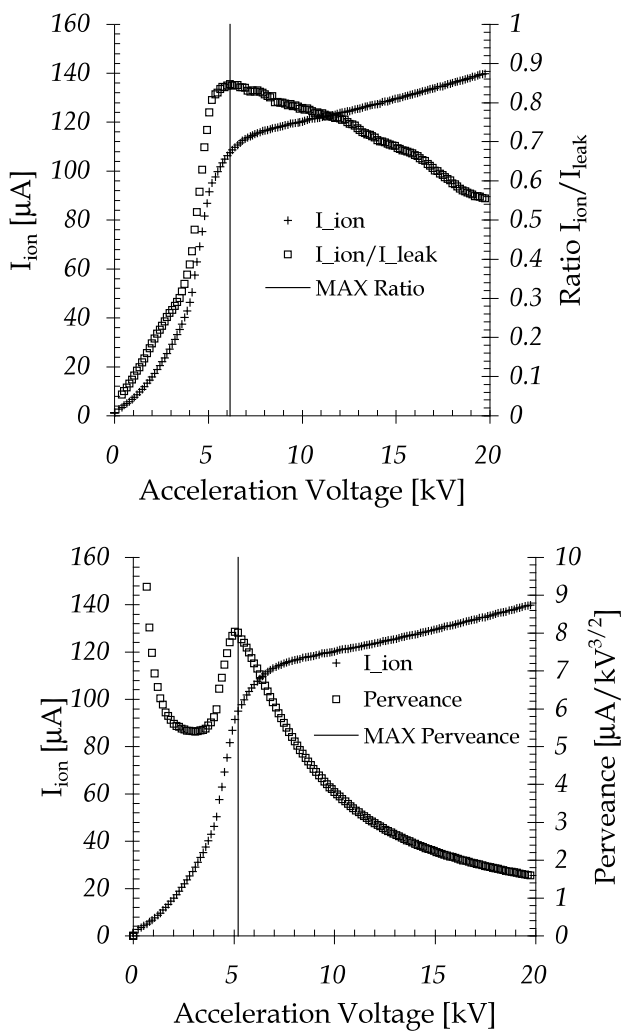

FIGURE 5. At the top figure the ratio of the extracted ion current to the accelerator power supply's leak current is plotted as a function of the acceleration voltage. The maximum of the ratio is close to the saturated value of the 
extracted ion current. At the right the perveance of the ion beam is plotted as a function of the acceleration voltage. The acceleration voltage corresponding the local maximum of the perveance is usually used as a voltage value for the saturated ion current.

The estimations of the saturation current for all three methods (linear fit, perveance-method and ratiomethod) for three different cases of extracted source current density are tabulated in the table 1 . Three different cases are: low $\left(\sim 0.5 \mathrm{~mA} / \mathrm{cm}^{2}\right)$, middle $(\sim 2.0$ $\left.\mathrm{mA} / \mathrm{cm}^{2}\right)$ and high $\left(\sim 4.5 \mathrm{~mA} / \mathrm{cm}^{2}\right)$ current density.

\begin{tabular}{r|c|cc|cc} 
& \multicolumn{2}{c|}{ Fit } & \multicolumn{2}{|c|}{ Perveance } & \multicolumn{2}{c}{ Ratio } \\
Current Density & $\mathrm{I}_{\text {ion }}[\mu \mathrm{A}]$ & $\mathrm{I}_{\text {ion }}[\mu \mathrm{A}]$ & from Fit & $\mathrm{I}_{\text {ion }}[\mu \mathrm{A}]$ & from Fit \\
\hline Low & 44.4 & 40.1 & $90 \%$ & 42.9 & $97 \%$ \\
Middle & 111.3 & 91.5 & $82 \%$ & 107.6 & $97 \%$ \\
High & 314.6 & 262 & $83 \%$ & 320.5 & $102 \%$
\end{tabular}

TABLE 1. Comparison between the perveance or the ratio method to the fit method. The percentage of the current estimated using the perveance and the ratio method from the fit method are shown also. The local maximum of the perveance gives systematically lower values for the current.

\section{B. Beam Emmitance}

A pepper-pot emittance measurement device was used for the beam emittance measurements. In the beam emittance measurements, the beam travelled through a discriminator, which is a plate with many tiny holes. A film holder was located behind the discriminator. The beam exposed a pattern on a film. From this pattern, the emittance of the beam at the discriminator position can be calculated. The discriminato is a $200 \mu \mathrm{m}$ thick tantalum plate. Holes with $200 \mu \mathrm{m}$ in diameter were drilled in the plate in a star-type pattern.

The pepper-pot device measures the $r r$ ' emittances. The value can be calculated using the known distances and dimensions of the measurement set-up (see figure 6) using the following equations:

$$
\begin{aligned}
& r=h \\
& r^{\prime}=\frac{d r}{d z}=\frac{H-h}{d} \\
& \Delta r^{\prime}=s
\end{aligned}
$$

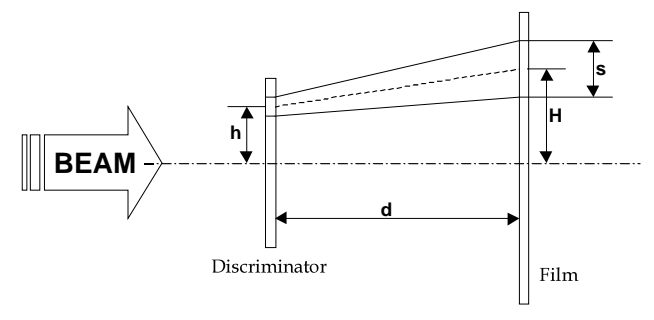

FIGURE 6. The variables used to calculate the beam emittance in the equation.

A microscope was used to measure the dot sizes and the distances between the dots on the film. From these numbers, $r$ and $r$ ' values are calculated. The actual emittance value is obtained from the $r, r$ ' values by fitting an ellipse function around the measured points. The analytical formula for an ellipse is[5]

$$
r^{\prime}= \pm \frac{B}{A} \sqrt{A^{2}-r^{2}}+C r+D
$$

where the A, B, C and D are constants. The 'divergence' term $(\mathrm{Cr}+\mathrm{D})$ can be obtained by fitting a linear function to the $r$ and r'values. The constants $A$ and $\mathrm{B}$ are the width and the length of the ellipse. The rr' emittance is then given by

$$
\varepsilon_{r r^{\prime}}=\pi A B
$$

\section{Ion Source Gas Efficiency}

The source gas efficiency was measured using argon gas and a calibrated leak. The stainless-steel capillary tube type leak was made by VTI (Vacuum Technology Inc.). It is an open-end type leak, which requires an external gas bottle. The manufacturer calibrates the leak using three input gas pressures. The leak was calibrated only for argon gas. The input pressure was monitored using a high pressure gauge in the input gas line of the leak and with the high pressure regulator at the gas bottle. The error in the leak rate is $\pm 15 \%$.

The gas efficiency is obtained by determining the total ion beam current and dividing it by the total number of neutral particles, flowing to the source. The part of the neutrals which are not ionized are being evacuated to the vacuum pumps. The gas efficiency for single charged, atomic gas can be written: 


$$
\eta_{g}=\frac{I_{i o n}}{e Q_{n} n_{0}}
$$

where the $\left[I_{i o n}\right]=\mathrm{A},\left[Q_{n}\right]=\operatorname{sccs}$ and $n_{0}=2.687 \times 10^{19}$ $\mathrm{cm}^{-3}$ is the Loschmidt's constant.

\section{RESULTS}

\section{A. Ion Current Density}

In figure 7 the argon ion current density is plotted as a function of source pressure for six different microwave powers. The pressure is measured using an ion gauge. The measurements are performed up to the maximum power in each pressure. In the case of low pressure and high microwave power the plasma becomes unstable.

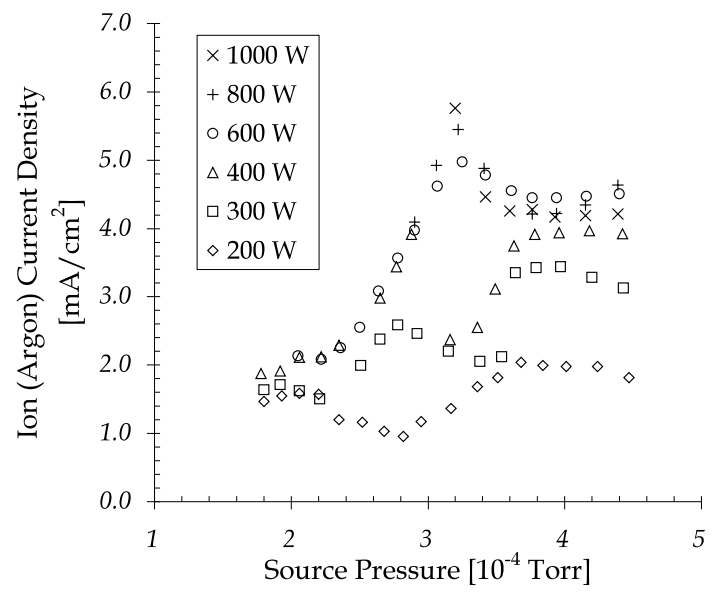

FIGURE 7. The ion current density of the microwave source measured at different pressures of argon gas. Three distinctive zones can be noticed, a low plasma density zone ( $\mathrm{p}<2 \mathrm{e}-4$ Torr $)$, transition zone $(2 \mathrm{e}-4<\mathrm{p}<4 \mathrm{e}-4$ Torr $)$ and high plasma density zone ( $\mathrm{p}>4 \mathrm{e}-4$ Torr).

Three regimes can be distinguished in the current density plot. In the first region the dependence of the current density to the microwave power is small. High power cannot be used at all, because plasma becomes unstable. In the second region a transition takes place.
Highest current densities are obtained in this region. Then the current is saturating in the third region. In this region the current density increases with increasing microwave power until it reaches saturation at $\sim 600 \mathrm{~W}$

The microwave source was also operated by using two frequencies simultaneously: $2.45 \mathrm{GHz}$ microwaves and $13.56 \mathrm{MHz}$ RF frequency. Microwave was coupled to the source using the existing coaxial coupler, and for the $13.56 \mathrm{MHz}$ a small antenna was used. The $13.56 \mathrm{MHz}$ power was coupled to the source via a matching network, which matched the amplifier's $50 \mathrm{Ohm}$ impedance to the plasma's 1-2 Ohm impedance. See figure 8 for the picture of the measurement set-up.

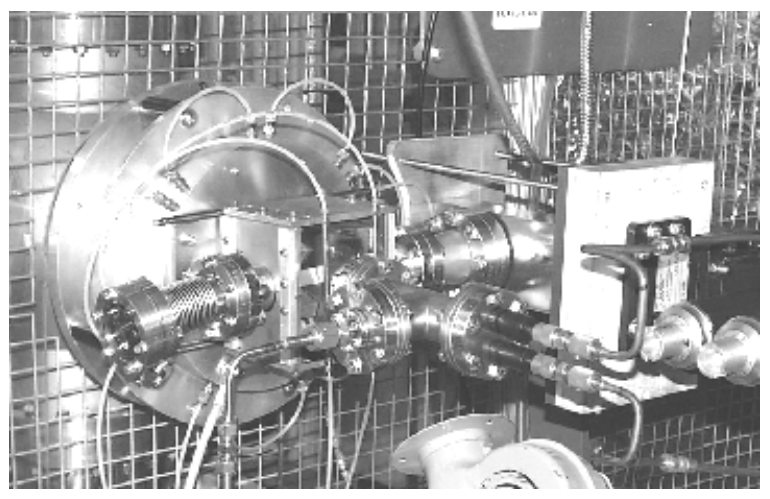

FIGURE 8. The microwave source is mounted to the test stand with side feed coaxial coupler for the microwaves and $\mathrm{RF}$ feed from the rear on the source.

In this type of compact microwave ion source, the microwave ionizes the gas at fairly high pressure region $\left(1-8 \times 10^{-4}\right.$ Torr $)$. The microwave operation and the RF-plasma operation are conveniently 'overlapping' at this pressure range. Significant enhancement for the plasma density can be obtained by operating the source simultaneously using both frequencies. The gain achieved with this dual frequency operation can be seen in figure 9, where the gain in current is plotted as a function of the RF power and source pressure using 400 watts of microwave power.

The double frequency operation is useful for both the microwave source and for the RF source. If a fast rise time of the plasma or good gas efficiency is required from the RF-source, microwave discharge could be used to provide the necessary background electrons to operate the RF source at low pressure range. Many times, RF sources have filament or laser 
driven photo cathodes to provide the background electrons for the discharge at low pressure. Microwave discharge would have the advantage of being a 'clean' source of electrons, no tungsten evaporation from the filaments or material from the photo cathode in the plasma. On the other hand, RF boosting can help the microwave discharge to work at higher pressure levels which would mean higher current density in a stable mode of operation. The RF-antenna could be made out of metals like, aluminum, copper or titanium. By inserting the antenna into the microwave discharge, some metallic ions could be produced by sputtering the surface of the antenna.

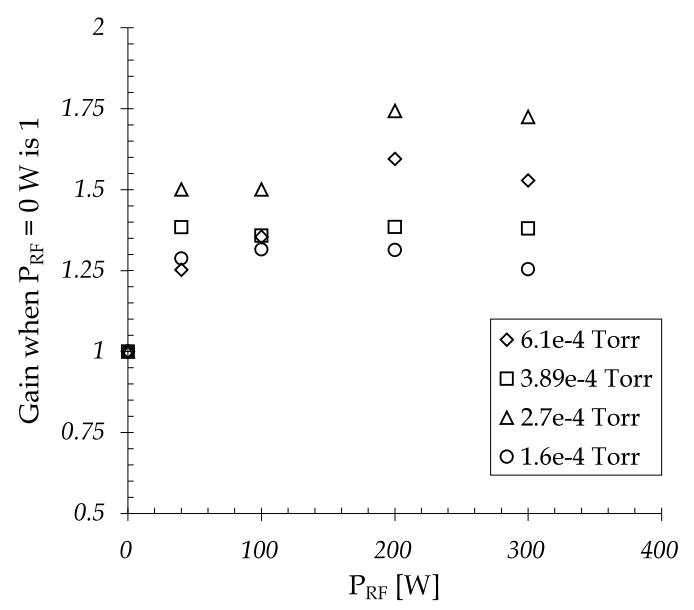

FIGURE 9. At $400 \mathrm{~W}$ of microwave power, the current gain is plotted as a function of the RF power and the source pressure. Note that every RF power level gives some gain for the extracted current. The gain is smallest at the low pressure level, increasing to the higher pressure level. The $2.7 \mathrm{e}-4$ Torr measurement is at the 'transition' pressure range of the source (see figure 7). That pressure range gives the highest gains.

\section{B. Charge State Distribution}

The microwave source has fairly high neutral gas density $\left(10^{13} \mathrm{~cm}^{-3}\right)$, compared to the plasma density (maximum in the order of $10^{12} \mathrm{~cm}^{-3}$ ). The electrons are not confined to the hot electron region in the ECR resonance layer and the electron energy is less than $100 \mathrm{eV}$. Despite those facts, the microwave source is able to produce multiply-charged ions. The charge state distribution for argon, oxygen and xenon are shown in figures 10-12 respectively.

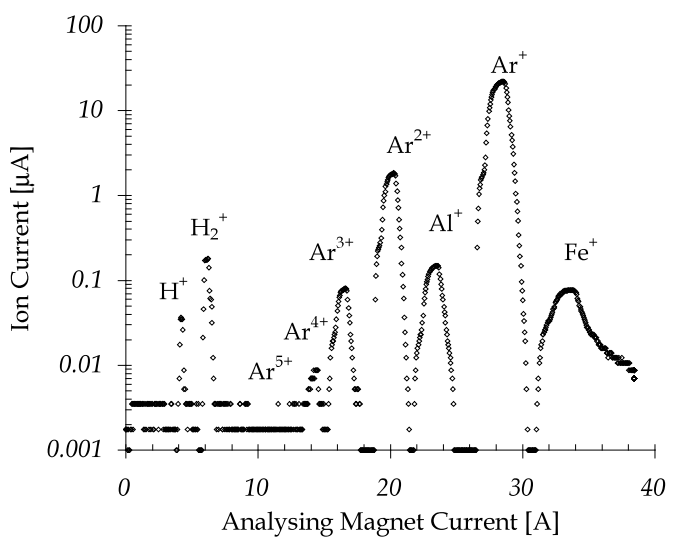

FIGURE 10. Argon spectra. High percentage of aluminum and iron in the spectra are due to sputtering of the front plate of the source (aluminum) and the middle electrode of the coaxial microwave feeder (steel). The sputtering is especially strong at high microwave power, in this measurement the microwave power was $1 \mathrm{~kW}$. The current for $\mathrm{Ar}^{5+}$ is at the lower limit of the measurement set-up.

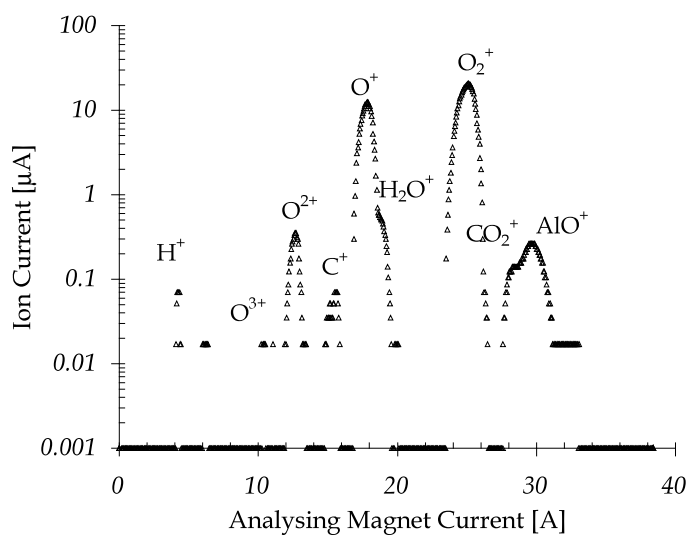

FIGURE 11. Oxygen spectra. Oxygen easily forms compounds with carbon atoms and hydrogen molecules. The microwave power was $400 \mathrm{~W}$. 


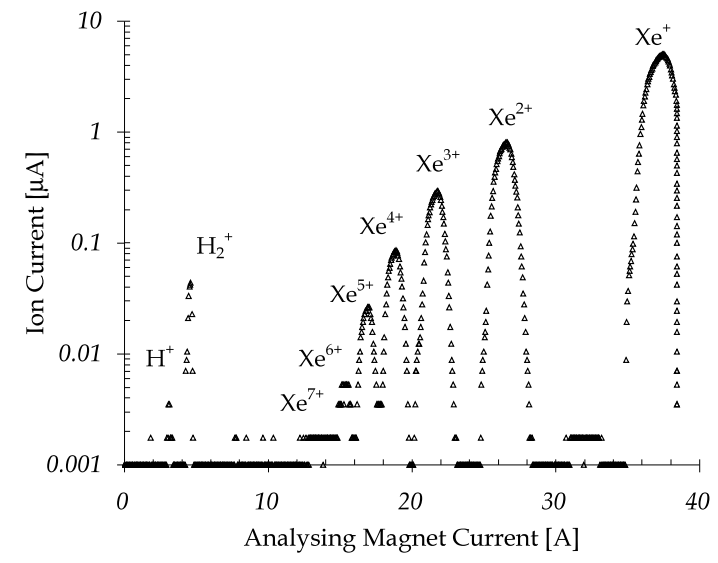

FIGURE 12. Xenon spectra. Different charge-states all the way to the $\mathrm{Xe}^{7+}$, are distinctly visible.

\section{Source Gas Efficiency}

The gas efficiency follows the trend similar to current density. There is a certain peak value for the current density, similarly to the gas efficiency. That peak is shifted to higher leak rates for increasing power.

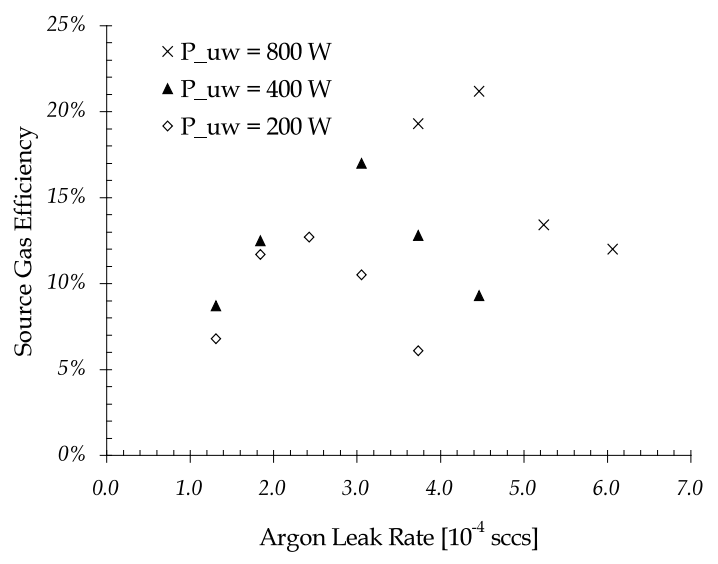

FIGURE 13. Gas efficiency measurement of the microwave source. The source efficiency looks similar to the current measurement, by increasing to a maximum value and then decreasing to a lower saturation. This can be observed especially in the high $(800 \mathrm{~W})$ power case. The pressure in the source is between $1.5 \mathrm{e}-4-6.0 \mathrm{e}-4$ Torr, depending on the leak rate.

\section{Beam Emittance}

An asymmetrical beam spread was noticed. This spread is probably due to the strong magnetic field close to the extraction aperture. When ions in the plasma are crossing the field, their trajectories are bended depending on their perpendicular velocity component to the magnetic field. There is no spread in the parallel direction to the field, except the spread due to the temperature distribution of the ions.

The pepper-pot method measures the $r r^{\prime}$ emittances. Although the $r r$ ' emittances are normally cylindrically symmetric, in this case, it is useful to calculate the emittance of the beam in two direction: perpendicular and parallel to the magnetic field. The ion temperature can be estimated from the size of the $r$ $=0$ dot in the parallel direction.

The emittance was also calculated, using simulation code IGUN. It is possible to include the ion temperature directly to the IGUN simulation using $T I$ parameter, but the code calculates the plasma meniscus incorrectly, leading to a hollow beam. A new method of including the ion temperature to the simulation has been developed for the SNS (Spallatation Neutron Source) $\mathrm{H}^{-}$source simulations and it is described in detail elsewhere[6].

The source parameters for the analyzed exposure are given in the table 2 . The electron temperature and current density was estimated using previous measurements. The current was not measured during the exposure. The ion temperature was given by the pepper-pot measurements. The parallel direction measurement was used. The phase space distribution of the trajectories for the simulation are shown in figure 14 .

\begin{tabular}{cccccc}
$\begin{array}{c}\mathbf{P}_{\mu \mathrm{w}} \\
{[\mathrm{W}]}\end{array}$ & $\begin{array}{c}\mathbf{p}_{\text {source }} \\
{\left[10^{-4} \text { Torr }\right]}\end{array}$ & $\begin{array}{c}\mathbf{U}_{\text {acc }} \\
{[\mathrm{kV}]}\end{array}$ & $\begin{array}{c}\mathbf{j} \\
{\left[\mathrm{mA} / \mathrm{cm}^{2}\right]}\end{array}$ & $\begin{array}{c}\mathbf{T}_{\mathbf{e}} \\
{[\mathrm{eV}]}\end{array}$ & $\begin{array}{c}\mathbf{T}_{\mathbf{i}} \\
{[\mathrm{eV}]}\end{array}$ \\
\hline 400 & 2.7 & 13 & $\sim 2.3$ & $\sim 15$ & $\sim 0.4$ \\
\hline
\end{tabular}

TABLE 2. Source operation parameters and estimated plasma parameter values for the simulation of the analysed pepper-pot measurement. 


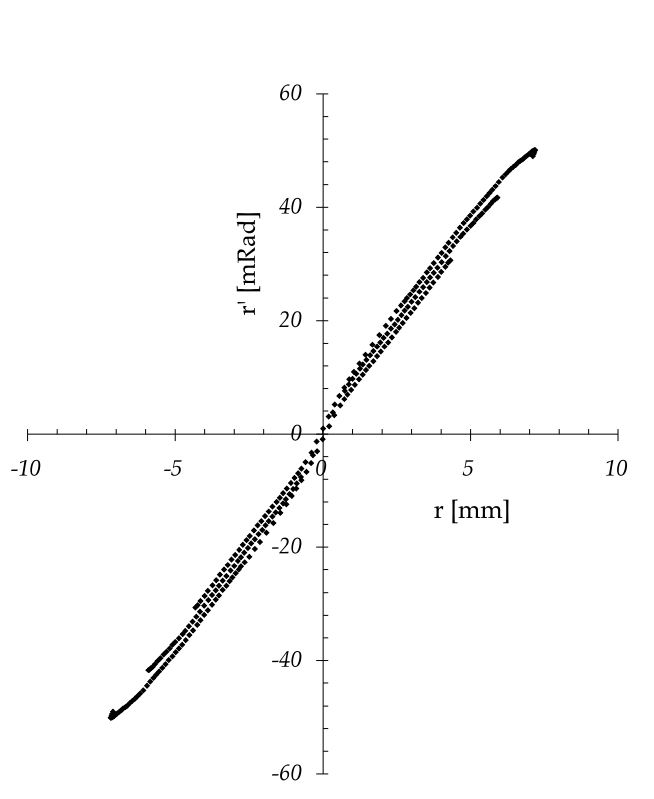

FIGURE 14. The rr' distribution of trajectories. Very small aberrations in the beam. Even with the finite ion temperature the 'width' of the distribution is small.

The rms-emittance was calculated using the simulation data. The $\varepsilon_{1 r m s}$ and $\varepsilon_{4 r m s}$ ellipses given by the parameters $\alpha, \beta$ and $\gamma$ of the rms distribution are plotted in figure 15. In the same figure, the measured emittance-ellipse-fits for the parallel and perpendicular directions are shown. The emittance values of the above four cases are tabulated in the table 3 .

\begin{tabular}{cccc}
\multicolumn{2}{c}{ Measured } & \multicolumn{2}{c}{ Simulation } \\
$\varepsilon_{\mathbf{n}, \mathbf{p e r}}$ & $\varepsilon_{\mathbf{n}, \mathbf{p a r}}$ & $\varepsilon_{\mathbf{n}, \mathbf{1 r m s}}$ & $\varepsilon_{\mathbf{n}, \mathbf{4 r m s}}$ \\
{$[\pi$-mm-mRad $]$} & {$[\pi$-mm-mRad] } & {$[\pi$-mm-mRad $]$} & {$[\pi$-mm-mRad $]$} \\
\hline 0.087 & 0.032 & 0.006 & 0.024 \\
\hline
\end{tabular}

TABLE 3. The normalized emittance values for the cases of measurement and simulation.

The agreement between the measurement and the simulation is fairly good. The comparable values of measured parallel emittance and the 4rms simulated value are in good agreement. If the temperature is not taken into account in the simulation the 4rms normalized value is $1.7 \times 10^{-3} \pi-m m-m R a d$, more than one order of magnitude smaller.

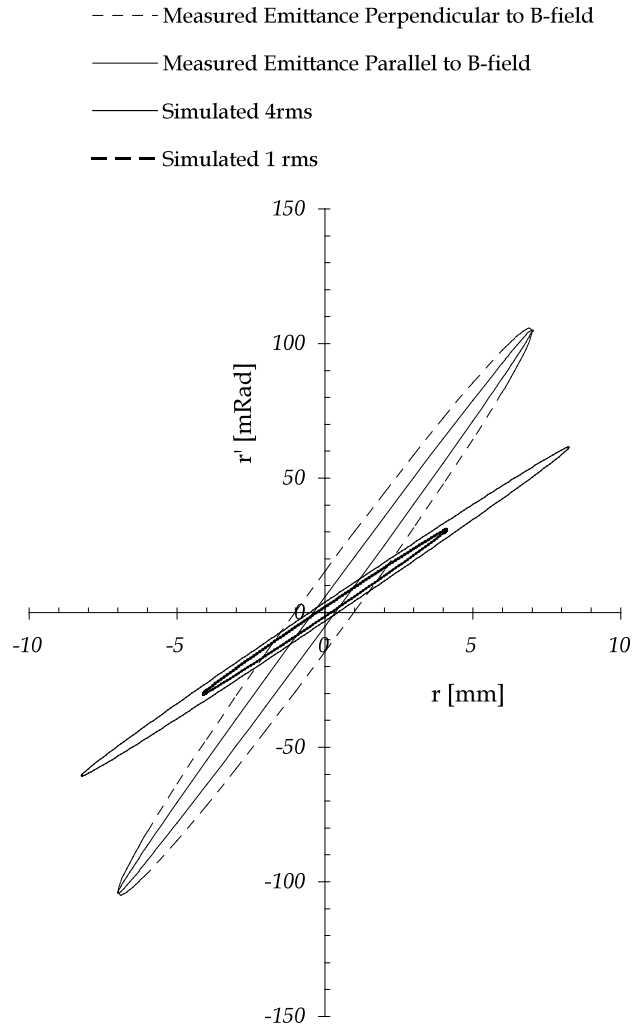

FIGURE 15. The emittance ellipses obtained by pepper-pot measurements and simulations.

\section{DISCUSSION}

The output current density (3-6 $\mathrm{mA} / \mathrm{cm}^{2}$ ) of the microwave ion source is (depending on the source pressure and power) about a factor of 2-3 higher than achieved with the conventional $2.45 \mathrm{GHz}$ ECR ion source. Some interesting conclusions of the wave/plasma interaction can be noted from the current density dependence of the power and pressure. These type of microwave sources operate at $2.45 \mathrm{GHz}$, which do not use the minimum $\mathrm{B}$ field (magnetic bottle) configuration like the conventional ECR ion sources, has the possibility to operate in the over-dense mode. In this mode the plasma density is increasing beyond the cut-off density and is limited only by the skindepth effect of the plasma. Skin-depth is in the case of the $2.45 \mathrm{GHz}$ is larger than the dimensions of the plasma, so that effective coupling of the microwaves can occur. The plasma density can still be increased after the cut-off density by even some orders of magnitude. The explanation for the non-linear behavior of the current density as a function of the source pressure might be the transition of the plasma density to the over-dense mode. The restricting factor for further increasing of the pressure (and thus the plasma density) in the source is the plasma formation 
in the coaxial feed system. In this high pressure region, plasma is ignited in the coaxial feed. This leads to strongly decreasing plasma density in the source.

A boost for the current density was achieved using 13.56 $\mathrm{MHz} R F$-frequencies in the source simultaneously with the microwaves. No coupling between the two frequencies were noted. The effect of the $R F$ on the current density was positive, when the source pressure was increased. This shows that the most efficient operation range for the $R F$ was not yet achieved at the typical microwave source pressure (low $10^{-4}$ Torr) range. Nevertheless the $R F$-boosting can be used in the microwave source to enhance boost the output current. On the other hand, the microwaves could be used in the $R F$-source to supply background electrons for the low pressure $R F$-operation.

The current values were measured and logged using a computer controlled data acquisition system. This will enable one to record various parameter during each sweep of the acceleration voltage. The saturation point was then determined from the maximum value of the ratio of the ion current over the accelerator voltage power supply's leak current. This method provides reasonable accurate estimates of the saturation current.

The low charge states in the beam are due to three different factors: fairly low electron energy, short confinement time of the ions to the hot electron zone and high neutral gas pressure. In the microwave source, the electron temperature in the electron cyclotron resonance layer is probably higher than the estimated values. But with fairly high neutral gas pressure, the high charge state ions are quickly recombined. For low charge states ion beam production, the microwave source is comparable to the conventional $2.45 \mathrm{GHz}$ ECR ion source. Although the percentage of the $2^{+}$ions compared to the $1^{+}$ion is less in the microwave source, with the higher current density, the current for the $2^{+}$ions is comparable.

The gas efficiency was measured using a calibrated leak. The leak was calibrated only for one gas, argon and no buffer gas was used in the measurement. The use of background plasma will increase the efficiency, especially at the low leak rates, because the source can still be operated at the optimum discharge conditions. The argon gas efficiency was in the order of $15 \%$ $25 \%$, depending on the microwave power and source pressure.

The emittance of the argon beam was measured using a pepper-pot measurement device. Kapton was used as a film material. Beam was noted to have a asymmetrical spread in the direction perpendicular to the dipole magnetic field of the source. This spread is related to $\mathbf{v} \times \mathbf{B}$ force turning the trajectories of ions having different thermal energy to different paths. In the parallel direction to the magnetic field, the spread was not present. The normalized, $r r^{\prime}$ emittancies using an extraction aperture of $3 \mathrm{~mm}$ in diameter and accelerator voltage of $13 \mathrm{kV}$, were 0.032 and $0.087 \pi$ $m m$-mrad for the parallel and perpendicular directions to the magnetic field respectively.

The emittance values were reproduced by simulations, using a novel method of introducing finite ion temperature to the beam. In this method the beam is extracted having zero temperature and the temperature is introduced to the beam after couple of hundred volts of acceleration. This way the plasma meniscus is formed properly. The simulation gives a normalized, 4rms emittance value of $0.024 \pi$ - $\mathrm{mm}$ mrad. This compares fairly well with the measured value of $0.032 \pi$-mm-mRad.

To address the problems of igniting a plasma in the coaxial feed, when operating in high pressure, a new microwave design is proposed. In that design, the microwaves are introduced to the source directly from the wave-guide through a window. Further enhancements include an iron yoke with a hole, going in front of the source to reduce the field strength at the extraction region to minimize the perpendicular spread of the emittance of the beam. Improvements in the current density and coupling of the microwaves to the plasma are being expected.

\section{ACKNOWLEDGMENTS}

This Work was supported by Department of Energy under Contract No. DE-AC03-76SF00098. One of authors (J. Reijonen) wants to acknowledge the support from the University of Jyväskylä, Finland. 


\section{REFERENCES}

[1] Dexter Magnetic Technologies, Magnetic Design, p. 28 (1998)

[2] D. Wutte, M. Leitner, M. Hoff, K. N. Leung, R. Gough, Design of a $2.45 \mathrm{GHz}$ ECR Ion Source for Production of Low and Medium Charge State Ions, $13^{\text {th }}$ ECR Workshop, Texas, 1997

[3] R. Becker and W. B. Herrmannsfeldt, Rev. Sci. Intrum. 63, 2756 (1992)

[4] J. Reijonen, et. al., Rev Sci. Instrum., 69, No. 2, p. 1138, (1998)

[5] R. Keller, et. al., IEEE Trans. Nucl. Sci., NS-32, p. 2579 (1985)

[6] J. Reijonen, R. Thomae, R. Keller, Evolution of the LEBT Layout for SNS, Proceedings of the $20^{\text {th }}$

Intern. LINAC Conf., Monterey, CA, August 21-

25, Ed. A Chao, SLAC R 561 (2001) 253 
This document was created with Win2PDF available at http://www.daneprairie.com. The unregistered version of Win2PDF is for evaluation or non-commercial use only. 